\title{
New and improved technology for manufacture of GMT primary mirror segments
}

\author{
Dae Wook Kim*a, b, James H. Burge ${ }^{\mathrm{a}}$, Johnathan M. Davis ${ }^{\mathrm{b}}$, Hubert M. Martin ${ }^{\mathrm{b}}$, Michael T. Tuell ${ }^{\mathrm{b}}$, \\ Logan R. Graves ${ }^{\mathrm{a}}$, Steve C. West ${ }^{\mathrm{b}}$ \\ ${ }^{a}$ College of Optical Sciences, University of Arizona, Tucson, AZ 85721, USA \\ ${ }^{\mathrm{b}}$ Steward Observatory, University of Arizona, Tucson, AZ 85721, USA
}

\begin{abstract}
The Giant Magellan Telescope (GMT) primary mirror consists of seven $8.4 \mathrm{~m}$ light-weight honeycomb mirrors that are being manufactured at the Richard F. Caris Mirror Lab (RFCML), University of Arizona. In order to manufacture the largest and most aspheric astronomical mirrors various high precision fabrication technologies have been developed, researched and implemented at the RFCML. The unique $8.4 \mathrm{~m}$ (in mirror diameter) capacity fabrication facilities are fully equipped with large optical generator (LOG), large polishing machine (LPM), stressed lap, rigid conformal lap (RC lap) and their process simulation/optimization intelligence called MATRIX. While the core capability and key manufacturing technologies have been well demonstrated by completing the first GMT off-axis segment, there have been significant hardware and software level improvements in order to improve and enhance the GMT primary mirror manufacturing efficiency. The new and improved manufacturing technology plays a key role to realize GMT, the next generation extremely large telescope enabling new science and discoveries, with high fabrication efficiency and confidence.
\end{abstract}

Keywords: Large optics, Computer Controlled Optical Surfacing (CCOS), Giant Magellan Telescope (GMT), Optical fabrication, Optics manufacturing

\section{INTRODUCTION}

\subsection{Richard F. Caris Mirror Lab overview}

The Richard F. Caris Mirror Lab (RFCML), a part of the Astronomy Department at the University of Arizona, is a unique facility which creates customized extremely large telescope mirrors with superior performance and quality all the way from glass chunks. The critical advantage of RFCML's large and fast (i.e. small f-number) mirrors is their lightweight honeycomb structure with excellent structural rigidity and thermal control capability. Its superb capability and technology enabled RFCML to produce some of the world's most powerful telescopes such as the $2 \times 8.4 \mathrm{~m}$ Large Binocular Telescope (LBT) ${ }^{1}$ and the $25 \mathrm{~m}$ Giant Magellan Telescope (GMT) ${ }^{2}$. As of 2016 the RFCML has cast five $6.5 \mathrm{~m}$ mirrors and seven $8.4 \mathrm{~m}$ mirrors including the first four of seven segments for the primary mirror of the GMT. Its test tower, in 2006, was replaced with a new $28 \mathrm{~m}$ tall tower containing four independent metrology systems in order to guide the GMT primary segments manufacturing process and perform the final test. The first $8.4 \mathrm{~m}$ off-axis GMT segment was successfully completed to an accuracy of $19 \mathrm{~nm}$ root mean square (RMS) error in 2012. ${ }^{3}$

\subsection{4 m primary segments for the Giant Magellan Telescope}

The GMT adopts a segmented optical system design, as shown in Figure 1 (left) to create extremely large optical aperture, which gives high diffraction limited resolution and superb light collecting efficiency. It has seven $8.4 \mathrm{~m}$ in diameter primary segments, the largest single mirrors that have been ever made, including six off-axis segments and one on-axis central segment.

*letter2dwk@hotmail.com

Advances in Optical and Mechanical Technologies for Telescopes and Instrumentation II, edited by Ramón Navarro, James H. Burge, Proc. of SPIE Vol. 9912, 99120P

(C) 2016 SPIE · CCC code: $0277-786$ X/16/\$18 - doi: 10.1117/12.2231911 
In addition to its large size, the main challenge to manufacture the segments comes from the highly aspheric shape of the off-axis segments shown in Figure 1 (right). More than $\sim 13 \mathrm{~mm}$ of aspheric departure requires customized metrology systems with complex null configurations and/or high dynamic range freeform metrology systems. Also, in order to fabricate the locally varying (i.e. locally freeform) optical surface, the fabrication technology needs to provide a freeform manufacturing capability such as adaptable tools and Computer Controlled Optical Surfacing (CCOS).
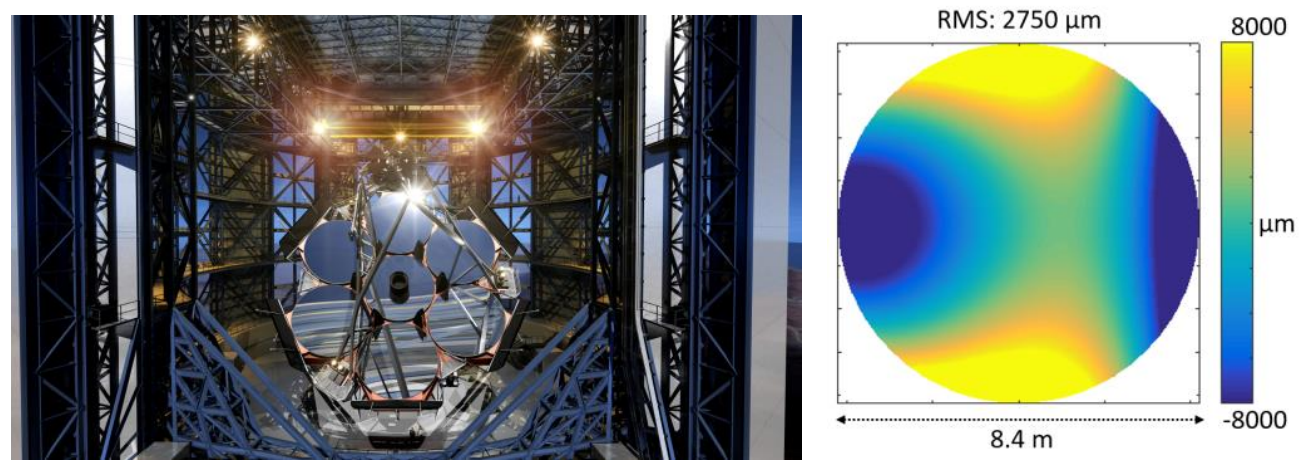

Figure 1. Artist image of the $25 \mathrm{~m}$ GMT with six $8.4 \mathrm{~m}$ off-axis segments and one $8.4 \mathrm{~m}$ on-axis central segment (left) ${ }^{4}$ and the aspheric departure map of the off-axis GMT segment when the parent vertex is located toward the left side of the map (right).

The optical design prescription of the off-axis GMT segment is presented in Table 1 . The primary segment has a near off-axis parabolic shape, and its aspheric departure from the best-fit sphere is mainly a $\sim 13 \mathrm{~mm}$ peak-to-valley astigmatic shape as shown in Figure 1 (right).

Table $1.8 .4 \mathrm{~m}$ honeycomb-sandwich GMT primary off-axis segment mirror optical design prescription ${ }^{5}$

\begin{tabular}{ccc}
\hline Optical parameter & Value & Note \\
\hline Radius of curvature & $36 \mathrm{~m}$ & Near parabola \\
Conic constant & -0.998286 & Distance from the parent vertex \\
Off-axis distance & $8.710 \mathrm{~m}$ & \\
Mirror diameter & $8.4 \mathrm{~m}$ & Astigmatic peak-to-valley departure \\
Aspheric departure & $\sim 13 \mathrm{~mm}$ & \\
\hline
\end{tabular}

\section{FREEFORM LARGE OPTICAL GENERATOR}

\subsection{Freeform Large Optical Generator overview}

The Large Optical Generator (LOG) shown in Figure 2 is one of the main fabrication machines executing diamond generating processes at the RFCML. The diamond wheel generating process creates an accurate optical surface shape with less than $\sim 10-20 \mu \mathrm{m}$ RMS error starting from a rough cast mirror blank from the spin-casting process. After the generating process is completed the mirror substrate goes through a grinding and polishing process. During the LOG generating process, converting a rotationally symmetric surface to the aspheric off-axis GMT segment shape, a metal (or resin) bound diamond wheel grinds the glass substrate at removal rates upwards of $1.5 \mathrm{~cm}^{3} / \mathrm{s}$ in order to remove $\sim 2400 \mathrm{~kg}$ from the front surface of the substrate. ${ }^{6}$ 


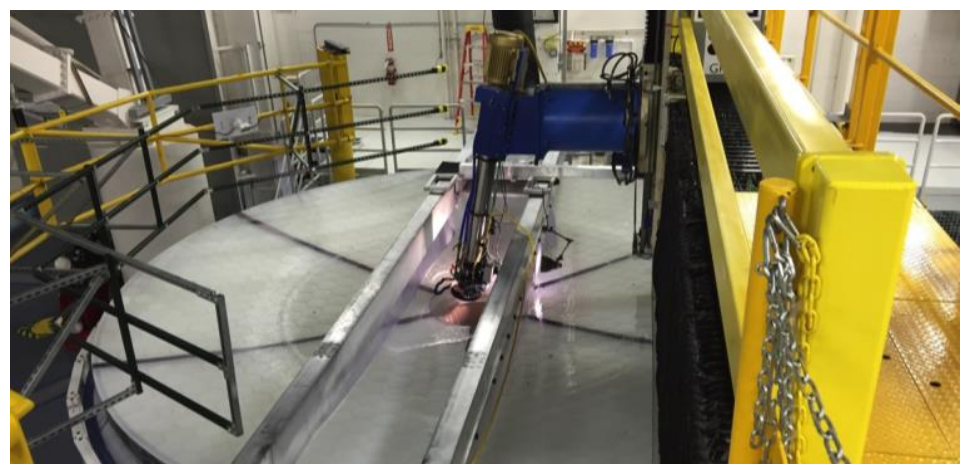

Figure 2. The $8.4 \mathrm{~m}$ class freeform LOG machine set up for the $6.5 \mathrm{~m}$ Tokyo Atacama Observatory (TAO) telescope primary mirror front surface generating process at the Richard F. Caris Mirror Lab, University of Arizona.

\subsection{Directed generating of an $8.4 \mathrm{~m}$ class freeform optical surface}

A three dimensional tool path control software for the freeform LOG has been successfully developed using the $\mathrm{SAGUARO}^{7}$ data processing platform. The software package offers various synthetic and/or measured target removal surface map processing capabilities in order to guide/control the precise motion of the generating wheel, for a directed generation of up to an $8.4 \mathrm{~m}$ freeform surface. The LOG machine's systematic or repeating motion error can be also evaluated and calibrated using the data processing and analysis functions in the software. The SAGUARO based LOG tool path control GUI (Graphical User Interface) is shown in Figure 3.

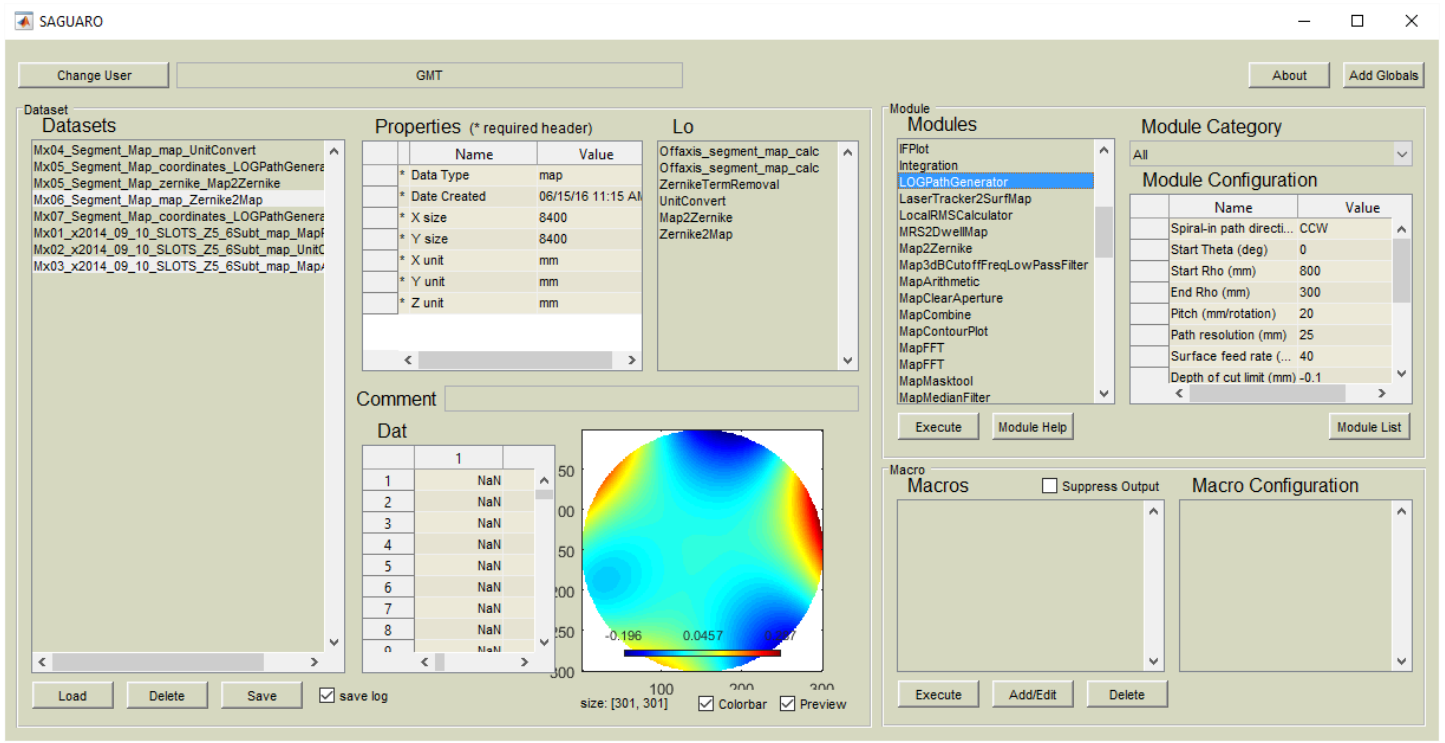

Figure 3. LOG tool path control software GUI based on the SAGUARO ${ }^{7}$ data processing platform. An exemplary LOG machine calibration map is shown in the middle preview section. Some of the tool path control parameters are shown in the top-right "Module Configuration" section.

Once the target error map from a measured/analyzed data and the machine calibration map are determined, the actual tool path considering various generating parameters such as cut-depth, feed rate, wheel diameter, and starting location (shown in the "Module Configuration" section in Figure 3) is created by executing a LOG tool path generating SAGUARO module. In addition to the normal directed generating mode, by applying advanced modules in the software pipeline, LOG is able to achieve special modes such as skipping unnecessary regions on the optical surface (e.g. very 
low areas) by actively lifting up the diamond wheel for a rapid maneuver. A tool path targeting a synthetic freeform surface generation with $\sim 0.5 \mathrm{~mm}$ of max height variation over the $8.4 \mathrm{~m}$ diameter substrate is presented in Figure 4.

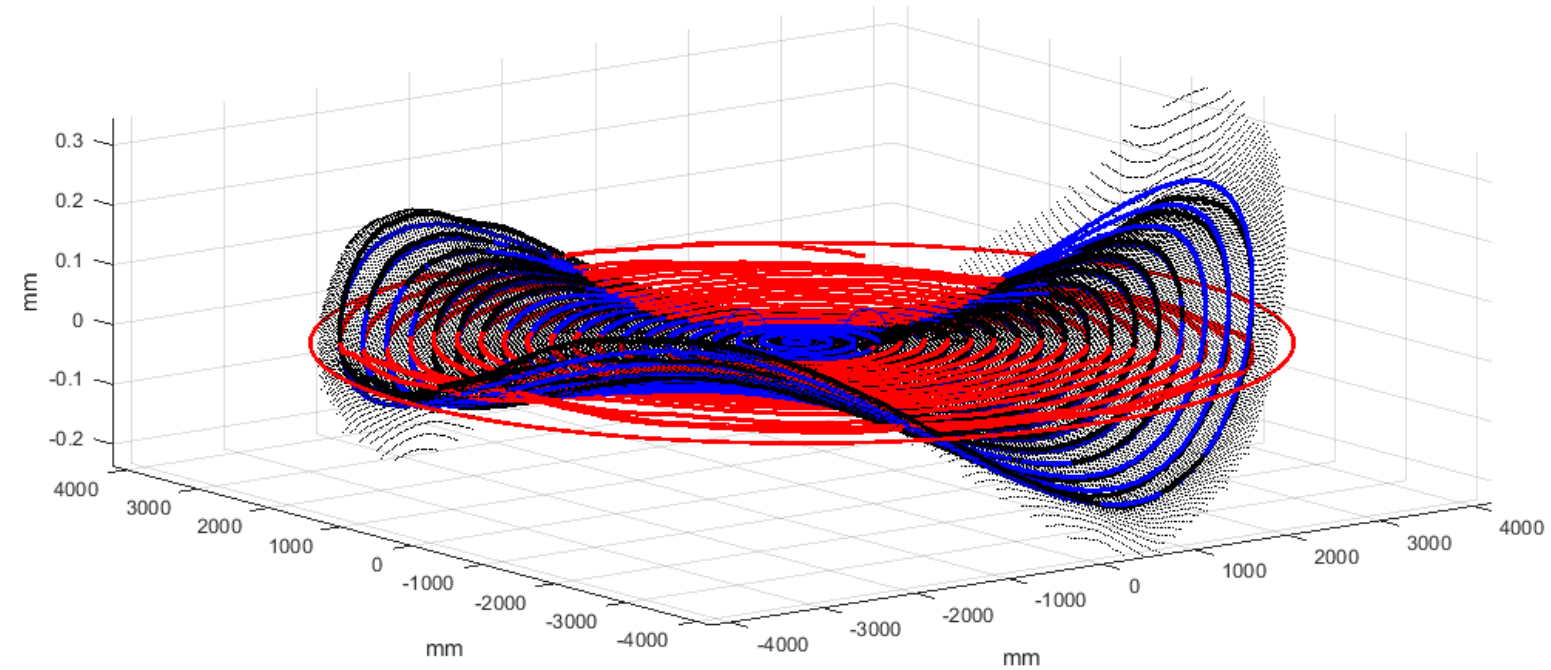

Figure 4. An $8.4 \mathrm{~m}$ freeform mirror surface generating tool path (black line) for a directed generating run considering both the LOG machine calibration (blue line) and the fine scale target removal map (red line). The black dots represents the full 3 dimensional surface map used to create the tool path. (Note: The feed rate, which defines the radial density of the spiral path, was adjusted to an unrealistically high value to show the tool path clearly in the figure.)

\section{$2.36 .5 \mathrm{~m}$ TAO primary mirror generating performance}

The LOG with directed generating capability has been used for generating the $6.5 \mathrm{~m}$ primary mirror substrate for the TAO (Tokyo Atacama Observatory) telescope shown in Figure 2. Two consecutive directed generating runs improved the surface radial profile RMS error from $48.6 \mu \mathrm{m}$ to $23.9 \mu \mathrm{m}$ to $10.4 \mu \mathrm{m}$ as presented in Figure 5. Over $50 \%$ improvement in terms of residual RMS error between each generating run clearly demonstrates the fast convergence rate of the enhanced LOG hardware and software performance.

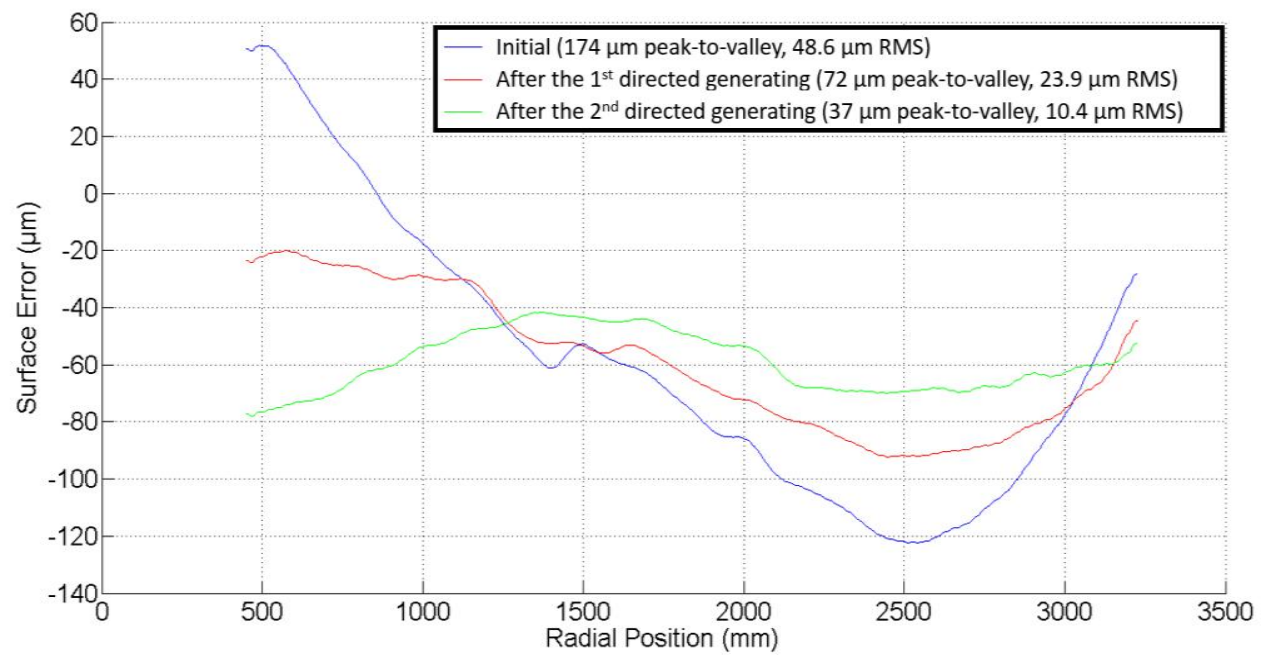

Figure 5. The $6.5 \mathrm{~m}$ TAO primary mirror substrate's radial profiles between two consecutive directed generating runs using the enhanced LOG machine and software demonstrate the fast convergence of the entire LOG process. 


\section{DUAL-HEAD LARGE POLISHING MACHINE}

\subsection{Dual-head Large Polishing Machine (LPM) overview}

The dual-head LPM with an improved simulation and optimization software MATRIX drives two polishing/figuring tools, an $1.2 \mathrm{~m}$ stressed lap and various sub-aperture $\left(\sim 5-60 \mathrm{~cm}\right.$ ) passive/RC (Rigid Conformal) laps ${ }^{8}$, simultaneously. The dual process was applied to the Large Synoptic Survey Telescope (LSST) ${ }^{9}$ monolithic primary-tertiary (two concentric mirror surfaces on a single glass substrate) mirror as shown in Figure 6. After the successful first implementation, the simultaneous dual mode has also been applied to a single optical surface case, the $8.4 \mathrm{~m}$ GMT offaxis segment (the 3rd segment out of the six off-axis segments) back surface fine grinding and polishing process.

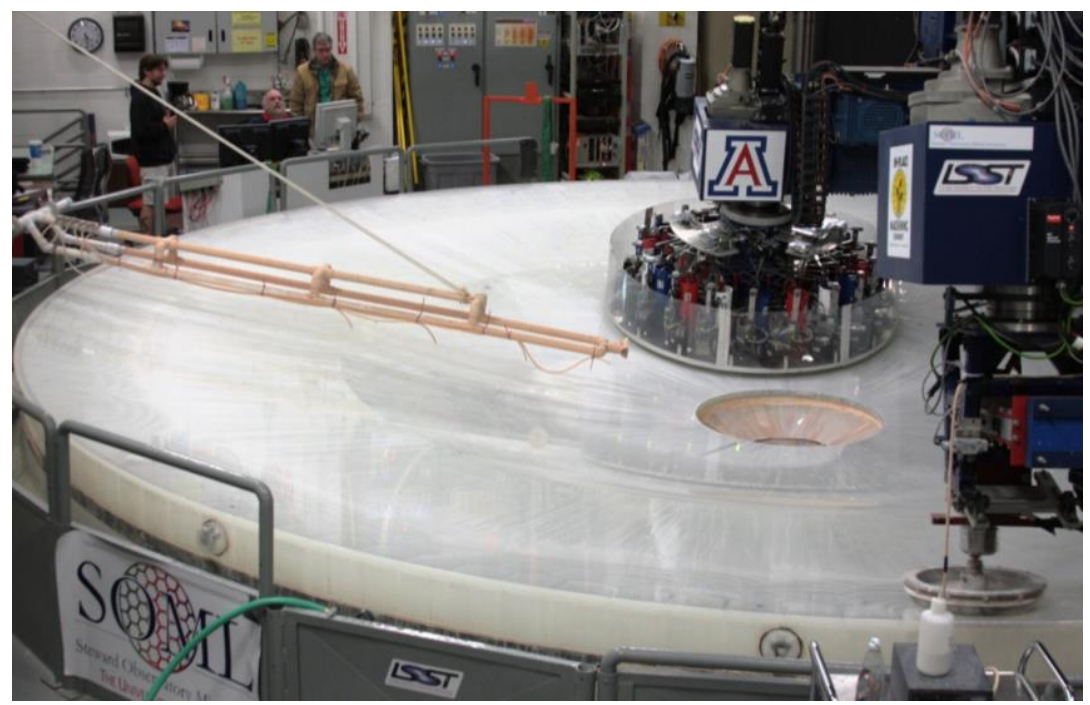

Figure 6. Dual-head $8.4 \mathrm{~m}$ class LPM with a $1.2 \mathrm{~m}$ stressed lap on the tertiary optical surface and a RC lap ${ }^{8}$ on the primary optical surface of the $\operatorname{LSST}^{9}$ primary-tertiary mirror at the Richard F. Caris Mirror Lab, University of Arizona. ${ }^{10}$

In order to fully utilize both tools on a single optical surface, the MATRIX simulates two tools with enhanced simultaneous optimization algorithms. The net effect of the two tool's removal maps matches or approaches the total target removal map on the single optical surface. Since the mirror's rotation speed variation, which usually controls the dwell time of a tool as a function of tool position on the mirror, is common for both tools, the optimization algorithm uses not only the dwell time, but additional parameters (e.g. tool stroke speed and tool pressure) to achieve an independent removal map from each tool from a single computer controlled optical surfacing run on the LPM.

\section{2 $1.2 \mathrm{~m}$ stressed lap with orbital tool stroke motion}

The $1.2 \mathrm{~m}$ (effective contact area diameter) stressed lap, which actively controls the tool shape using 18 real-time benders according to various local freeform surface shapes, has been upgraded with an orbital tool stroke motion capability. In addition to the original spin tool stroke mode, the upgraded stressed lap provides an orbital mode as depicted in Figure 7 (left). One of the most significant advantages of the orbital motion is the required shape change magnitude within a tool stroke motion. For instance, if a $1.2 \mathrm{~m}$ stressed lap on the $8.4 \mathrm{~m}$ GMT off-axis segment is located near the right-side edge (i.e. the center of the tool stroke motion: $12.61 \mathrm{~m}$ away from the parent vertex) in Figure 1 (right), the required shape change magnitude is only $33 \mu \mathrm{m}$ peak-to-valley for the orbital stroke mode as shown in Figure 7 (left) compared to the $1042 \mu \mathrm{m}$ for the spin stroke case (right). (Note: The orbital stroke radius $R_{\text {orbital }}$ was set to $150 \mathrm{~mm}$.) This greatly improves the active bending control accuracy as the target lap shape variation is very mild and slowly changing within a local tool stroking motion (e.g. 5 - 10 revolutions per minute orbital motion speed). 

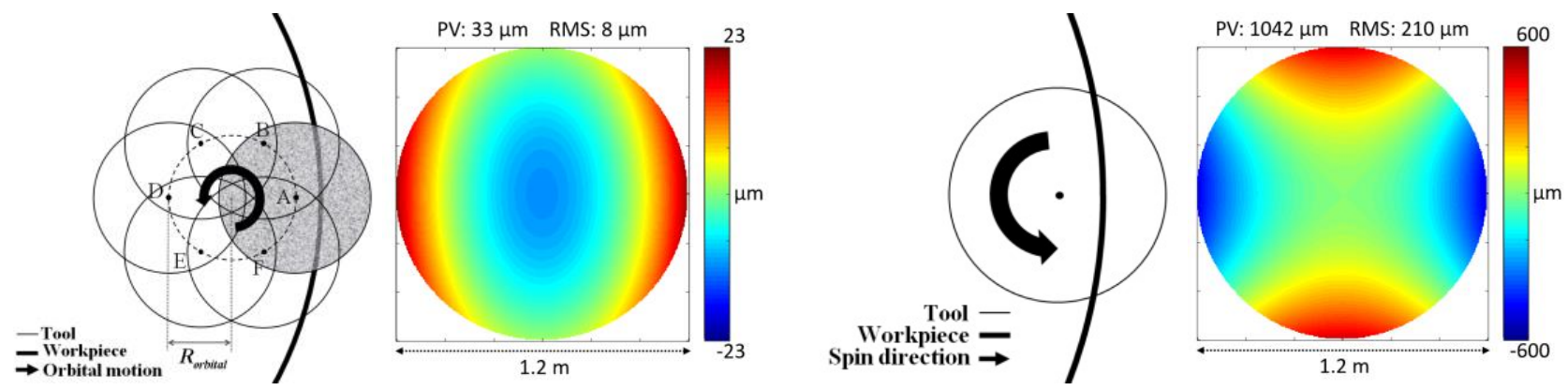

Figure 7. Two different types of tool stroke motions (left: orbital motion, right: spin motion) for the $1.2 \mathrm{~m}$ stressed lap and the required active lap shape change. The shape change map is the difference map between the position $\mathrm{A}$ and $\mathrm{D}$ for the orbital case (with $R_{\text {orbital }}=150 \mathrm{~mm}$ ) and between $90^{\circ}$ lap rotation for the spin case.

\subsection{Rapid fine grinding and polishing process using diamond pads}

A rapid fine grinding and polishing process using a Trizact bound-abrasive diamond pad has been developed and applied after a series of offline experiments checking the feasibility for the $8.4 \mathrm{~m}$ class optics application, which requires days of rubbing time, low subsurface damage ${ }^{11}$, large contact area between the pad and the optical surface, and a curved contact area to accommodate the large off-axis/freeform substrates such as the $8.4 \mathrm{~m}$ GMT off-axis segment. A part of the tiled Trizact pads during the curved surface polishing experiments is shown in the Figure 8. The area marked black in Figure 8 (left) represents the non-contact area in the central region during the pad conditioning phase. The hexagonal diamond pellets in Figure 8 (right) are $\sim 3 \mathrm{~mm}$ across the hexagons and $\sim 700 \mu \mathrm{m}$ thick with $\sim 10 \mu \mathrm{m}$ diamond particles in it.
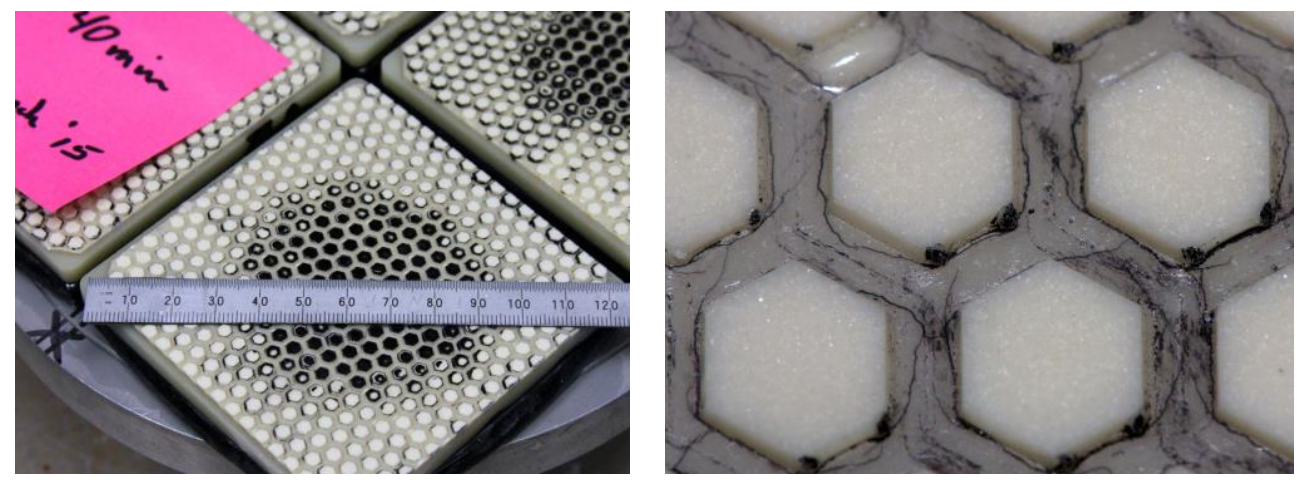

Figure 8. Trizact diamond pads with Pressure Sensitive Adhesive (PSA) is installed on flat plastic squares, attached to tool substrate with nominal pitch layer (left). A black marking was applied for a visual check of the contact area during the conditioning process on a curved surface with $\sim 8 \mathrm{~m}$ radius of curvature. A zoom-in view of the hexagonal diamond pellets with $\sim 10 \mu \mathrm{m}$ diamond particles is also presented (right).

The measured removal rates for the Trizact pads and the traditional loose abrasive grinding cases were measured, compared and presented in Table 2. The measured Trizact removal rate showed approximately a $1.5-2.5$ times faster process compared to the loose abrasive grinding process for a similar grinding particle size. (It should be noted that the absolute values may vary as a function of specific substrate materials, chemical effects and other factors.) 
Table 2. Removal rate comparison between Trizact diamond pads and the loose abrasive grinding process

\begin{tabular}{cccc}
\hline $\begin{array}{c}\text { Grinding } \\
\text { process type }\end{array}$ & $\begin{array}{c}\text { Grinding particle size } \\
(\boldsymbol{\mu m})\end{array}$ & $\begin{array}{c}\text { Glass } \\
\text { substrate }\end{array}$ & $\begin{array}{c}\text { Removal rate } \\
(\boldsymbol{\mu m} / \mathbf{p s i}(\mathbf{m} / \mathbf{s e c}) \text { hour })\end{array}$ \\
\hline \multirow{3}{*}{ Aluminum Oxide loose abrasive } & 25 & & $\sim 1500$ \\
& 12 & Pyrex & $\sim 650$ \\
Trizact diamond pad & 5 & & $\sim 170$ \\
& 20 & Pyrex & $\sim 2800$ \\
& 9 & & $\sim 400$ \\
\hline
\end{tabular}

The first Trizact pad process on an $8.4 \mathrm{~m}$ class optic has been successfully applied to the back surface fine grinding and polishing process for the 3rd GMT off-axis segment. The overall fine grinding and polishing-out duration has been shortened by a factor of $\sim 1.5$ compared to the traditional loose abrasive process.

\section{CONCLUDING REMARKS}

Since the first GMT off-axis segment was successfully completed ${ }^{3}$ in 2012 , the second, third off-axis segments and the on-axis center segment have been successfully cast. In order to manufacture the largest and most aspheric astronomical mirrors various high precision fabrication and testing technologies have been investigated, developed and implemented at the RFCML. The major fabrication system upgrades include LOG hardware and software improvement allowing $8.4 \mathrm{~m}$ in diameter freeform surface generation, rapid fine grinding and polishing process utilizing Trizact diamond pads, MATRIX software with an enhanced multiple tool optimization feature, and stressed lap with orbital tool stroke motion capability. These new and improved manufacturing technology will play a key role in the realization of GMT and other future extremely large optical systems.

\section{ACKNOWLEDGEMENTS}

This work has been supported by the GMTO Corporation, a non-profit organization operated on behalf of an international consortium of universities and institutions: Astronomy Australia Ltd, the Australian National University, the Carnegie Institution for Science, Harvard University, the Korea Astronomy and Space Science Institute, the Smithsonian Institution, The University of Texas at Austin, Texas A\&M University, University of Arizona, University of Chicago, and the Sao Paulo Research Foundation (FAPESP).

This material is based in part upon work performed for the "Post-processing of Freeform Optics" project supported by the Korea Basic Science Institute.

The SAGUARO data processing related software development is funded by the II-VI Foundation Block grant.

This material is based in part upon work supported by AURA through the National Science Foundation under Scientific Program Order No. 10 as issued for support of the Giant Segmented Mirror Telescope for the United States Astronomical Community, in accordance with Proposal No. AST-0443999 submitted by AURA.

\section{REFERENCES}

[1] J. M. Hill, et al., "The Large Binocular Telescope: binocular all the time", Proc. SPIE 9145, Ground-based and Airborne Telescopes V, 914502 (2014).

[2] R. A. Bernstein, et al., "Overview and status of the Giant Magellan Telescope project", Proc. SPIE 9145, Groundbased and Airborne Telescopes V, 91451C (2014).

[3] H. M. Martin, R. G. Allen, J. H. Burge, D. W. Kim, J. S. Kingsley, K. Law, R. D. Lutz, P. A. Strittmatter, P. Su, M. T. Tuell, S. C. West, P. Zhou, "Production of 8.4m segments for the Giant Magellan Telescope," Proc. SPIE 8450, Modern Technologies in Space- and Ground-based Telescopes and Instrumentation II, 84502D (September 13, 2012). 
[4] http://www.gmto.org/

[5] H. M. Martin, R. G. Allen, J. H. Burge, D. W. Kim, J. S. Kingsley, M. T. Tuell, S. C. West, C. Zhao and T. Zobrist, "Fabrication and testing of the first $8.4 \mathrm{~m}$ off-axis segment for the Giant Magellan Telescope," Proc. SPIE 7739, (2010).

[6] Johnathan M. Davis, Hubert M. Martin, Dae Wook Kim, Adrian R. Loeff, Kurtis L. Kenagy, Raymond W. Sisk, Jeffery R. Hagen, "Advances in diamond generating for 8.4 meter telescope mirrors," Proc. SPIE 9633, Optifab 2015, 96330Y (October 11, 2015).

[7] D. W. Kim, B. J. Lewis, and J. H. Burge, "Open-source data analysis and visualization software platform: SAGUARO," Proc. SPIE, (2011).

[8] Dae Wook Kim and James H. Burge, "Rigid conformal polishing tool using non-linear visco-elastic effect," Opt. Express. 18, 2242-2257 (2010).

[9] W. Gressler, J. DeVries, E. Hileman, D. R. Neill, J. Sebag, O. Wiecha, J. Andrew, P. Lotz and W. Schoening, "LSST telescope and site status", Proc. SPIE 9145, 91451A (2014).

[10] Buddy Martin and Dae Wook Kim, "How do you build a mirror for one of the world's biggest telescopes?," The Conversation (2016).

[11] James B. Johnson, Dae Wook Kim, Robert E. Parks, James H. Burge, "New approach for pre-polish grinding with low subsurface damage," Proc. SPIE 8126, Optical Manufacturing and Testing IX, 81261E (September 27, 2011). 